EPJ Web of Conferences 28, 12069 (2012)

DOI: $10.1051 /$ epjconf/20122812069

(C) Owned by the authors, published by EDP Sciences, 2012

\title{
ATLAS Upgrade for the HL-LHC: meeting the challenges of a five-fold increase in collision rate
}

\author{
Peter Vankov ${ }^{1, a}$ \\ Deutsches Elektronen Synchrotron, DESY, Notkestrasse 85, 22607 Hamburg, Germany \\ On behalf of the ATLAS collaboration
}

\begin{abstract}
With the LHC successfully collecting data at $7 \mathrm{TeV}$, plans are actively advancing for a series of upgrades leading eventually to about five times the LHC design-luminosity some 10-years from now in the High-Luminosity LHC (HL-LHC) project. Coping with the high instantaneous and integrated luminosity will require many changes to the ATLAS detector. The designs are developing rapidly for an all-new inner-tracker, significant changes in the calorimeter and muon systems, as well as improved triggers. This article summarizes the environment expected at the HL-LHC and the status of various improvements to the ATLAS detector.
\end{abstract}

\section{Introduction}

ATLAS [1], at the CERN Large Hadron Collider (LHC) [2], is a general-purpose experiment designed to explore the proton-proton $(p p)$ collisions at the LHC with center-ofmass energies of up to $\sqrt{s}=14 \mathrm{TeV}$ at a maximum luminosity of $\mathcal{L}^{\text {peak }}=10^{34} \mathrm{~cm}^{-2} \mathrm{~s}^{-1}$. The high collision energy along with the high luminosity at the LHC would eventually allow observation of new physics at the TeV scale. ATLAS is constructed to fully exploit the physics potential of the LHC, which includes the discovery of the Higgs particle, as well as searches for effects beyond the Standard Model (SM).

As illustrated in Fig. 1, ATLAS comprises three basic subsystems: the Inner Detector, ID (Pixel, SCT, TRT), housed in a solenoid creating a magnetic field of $2 \mathrm{~T}$, the Calorimetry system (Liquid Argon and Tile) and the Muon Spectrometer (MS) with its associated superconducting toroidal magnets supplying a magnetic field of $0.5 \mathrm{~T}$. A three-

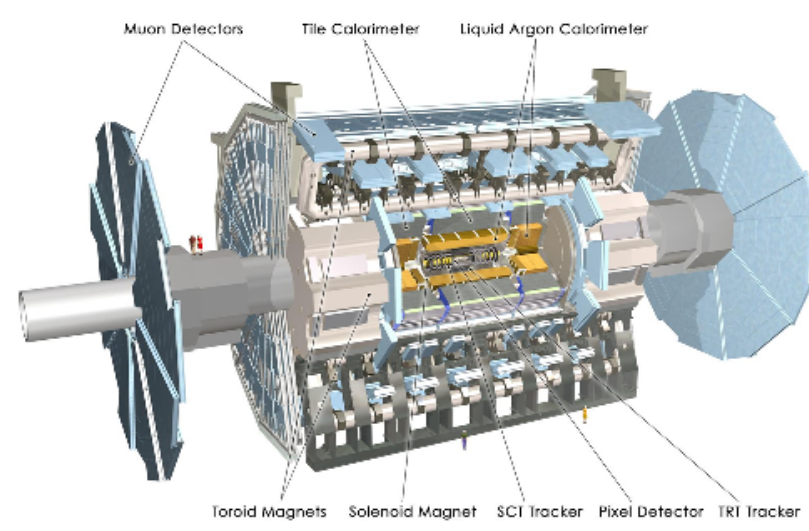

Fig. 1. The ATLAS experiment.

level trigger system is used to select the events of interest,

\footnotetext{
a e-mail: peter.vankov@cern.ch
}

providing a final average trigger-rate of a few hundred $\mathrm{Hz}$. The overall dimensions of $44 \mathrm{~m}$ in length and $25 \mathrm{~m}$ in diameter, make ATLAS the largest detector in collider experiments.

ATLAS has been successfully taking $p p$ collision data at $\sqrt{s}=7 \mathrm{TeV}(3.5 \mathrm{TeV}$ per beam) since March 2010 . As a result of the excellent performance and operation of the experiment, by the end of October 2011, ATLAS has recorded an integrated luminosity of $5 \mathrm{fb}^{-1}$ with stable beams, corresponding to an overall data-taking efficiency of $94 \%$.

In the next years, LHC will undergo a series of upgrades leading ultimately to five times increase of the instantaneous luminosity in the High-Luminosity LHC (HLLHC) project. The goal is to extend the dataset from about $300 \mathrm{fb}^{-1}$, expected to be collected by the end of the LHC run (in $\sim 2020$ ), to $3000 \mathrm{fb}^{-1}$ by $\sim 2030$. The foreseen higher luminosity at the HL-LHC is a great challenge for ATLAS. Meeting it will require significant detector optimizations, changes and improvements, which are subject of these proceedings.

\section{HL-LHC and the ATLAS Upgrade Plans}

The main motivation for the HL-LHC is to extend and to improve the LHC physics program [3]. Depending on the results from the LHC data, some of the physics problems that could be addressed at the HL-LHC are: measuring of the Higgs rare decays and Higgs self-couplings; performing a complete supersymmetry spectroscopy; searching (extending limits) for new gauge bosons $\left(W^{\prime}, Z^{\prime}\right)$; searching for a quark and lepton substructure.

The harsher radiation environment and higher detector occupancies at the HL-LHC imply major changes to most of the ATLAS systems, specially those at low radii and large pseudorapidity, $\eta$. A general guideline for these changes is maintaining the same (or better) level of detector performance as at the LHC. The ID, forward calorimeter and forward muon wheels will be affected the 
most by the higher particle fluxes and radiation damage, requiring replacement or significant upgrade, whereas the barrel calorimeters and muon chambers are expected to be capable of handling the conditions and will not be modified. New, radiation-hard tracking detectors with higher granularity and higher bandwidth, as well as radiation-hard front-end (FE) electronics are foreseen. The higher event rates and event sizes will be a challenge for the trigger and data acquisition (DAQ) systems, which will require a significant expansion of their capacity.

The ATLAS upgrade is planned in three phases, which correspond to the three long, technical shutdowns of the LHC towards the HL-LHC. Phase-O ( 24 months) will take place in 2013 and 2014 , the Phase-I ( 12 months) will be during 2018, and finally, the Phase-II ( 24 months) is scheduled for 2022-2023.

\section{ATLAS Upgrade: Phase 0}

The main objective of the 2013-2014 shutdown of the LHC is to perform interventions which will permit the machine to operate at its design parameters: center-of-mass energy of $\sqrt{s}=14 \mathrm{TeV}$ and luminosity of $1 \times 10^{34} \mathrm{~cm}^{-2} \mathrm{~s}^{-1}$.

ATLAS will use this two years period for detector consolidation works, including a new ID cooling system, a new neutron shielding of the MS, and a new beam pipe. The current beam pipe in the forward region is made of stainless steel which is a source of high backgrounds for the MS. The new beam pipe will be of aluminum, thus, reducing the backgrounds by $10-20 \%$.

The central ATLAS upgrade activity in Phase- 0 is the installation of a new barrel layer in the present Pixel detector, the so called IBL project.

\subsection{Insertable B-layer}

The Insertable B-layer (IBL) is an additional, $4^{\text {th }}$ pixel layer which will be inserted between the innermost pixel layer (the B-layer) and the beam pipe, as shown in Fig. 2, during the Phase-0 upgrade. To make the installation of the IBL possible, a new beam pipe in the central region, with reduced by $4 \mathrm{~mm}$ radius $(\mathrm{r}=29 \mathrm{~mm} \rightarrow \mathrm{r}=25 \mathrm{~mm})$, built of Beryllium, is envisaged.

As demonstrated in Ref [4], it is expected that the IBL will improve the vertex resolution, secondary vertex finding and b-tagging, hence extending the reach of the physics analysis. It will compensate for defects (irreparable failures of modules) in the existing B-layer, assuring tracking robustness. Moreover, IBL will help to preserve the tracking performance at the luminosity beyond $\mathcal{L}^{\text {peak }}$, e.g. in Phase-1, when the B-layer will suffer from radiation damage and high pile-up occupancies.

The baseline concept of the IBL consists of 14 staves, mounted directly on the beam pipe with a tilt angle of $14^{\circ}$. On each stave there are 16 to 32 modules depending on the sensor type. Currently, two silicon sensor types are under consideration: planar and 3D. The IBL modules will be equipped with a new readout chip, FE-I4 [5], which has been specially developed to function at high data transfer rates $(\sim 160 \mathrm{Mb} / \mathrm{s})$. The FE-I4 design allows an increase of the IBL segmentation by decreasing the pixel size from $50 \mu \mathrm{m} \times 400 \mu \mathrm{m}$ to $50 \mu \mathrm{m} \times 250 \mu \mathrm{m}$.

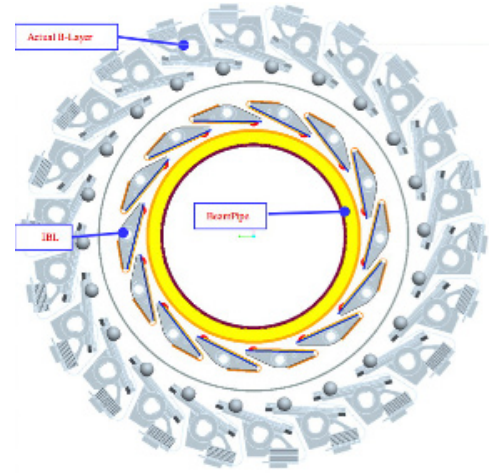

Fig. 2. Cross-section view of the current Pixel B-layer, the new beam pipe and the IBL.

\section{ATLAS Upgrade: Phase-I}

In 2018, the LHC will be stopped for an upgrade of the injectors and the collimators. Upgrade of the LINAC2 and increase of the Proton Synchrotron Booster output energy are planned. The data-taking will be resumed after one year shutdown with luminosity of $2 \times 10^{34} \mathrm{~cm}^{-2} \mathrm{~s}^{-1}$. During the shutdown, ATLAS intends to accomplish the second stage of its upgrade program, the Phase-I.

In Phase-I, installation of new Muon Small Wheels and introducing of new trigger schemes (Fast TracKer, topological triggers, improved L1Calo granularity) are proposed to handle luminosities well beyond the nominal values.

\subsection{New Muon Small Wheels}

A replacement of the first endcap station of the Muon Spectrometer, the Muon Small Wheel (MSW), built of Monitored Drift Tubes (MDT) and Cathode Strip Chambers (CSC), is proposed. The concern is that for luminosities $\mathcal{L}>\mathcal{L}^{\text {peak }}$, in addition to the higher number of pile-up events per bunch-crossing, large amounts of cavern background will be induced, affecting a large $|\eta|$ region of the MSW. The current system in this region will struggle badly to cope with this and therefore a replacement is required.

The new Muon Small Wheels must ensure efficient tracking at high particle rate (up to $\mathcal{L}=5 \times 10^{34} \mathrm{~cm}^{-2} \mathrm{~s}^{-1}$ ) and large $|\eta|$, with position resolution of $<100 \mu \mathrm{m}$. Furthermore, the new MSW will be integrated into the Level1 trigger [9]. Several detector technologies are under investigation at the moment: small diameter MDT's (sMDT) complemented with fast trigger chambers - Resistive Plate Chambers (RPC) or Thin Gap Chambers (TGC); Fine strip TGC's; Micro-MEsh GAseous Structures (MicroMEGAs); or some other combinations of these.

\subsection{New Trigger Schemes}

At Phase-I, more sophisticated triggers will be required. For this, the Fast TracKer (FTK) trigger project has been initiated [6]. At the FTK, the track finding and fitting are conducted at a hardware level, which makes it extremely fast. At the current ATLAS, this task is performed by the trigger Level-2 software farm. FTK will provide the track parameters at the beginning of the Level-2 processing. This 
way, the load on Level-2 will be diminished and extra resources will be available for more advanced selection algorithms, which ultimately could improve the b-tagging, lepton identification, etc.

Suggestions are also in place for combining trigger objects at Level-1 (topological triggers) and for implementing full granularity readout of the calorimeter. The latter will strongly improve the triggering capabilities for electrons and photons at Level-1.

\section{ATLAS Upgrade: Phase-II}

The ATLAS Phase-II upgrade is scheduled for 2022 and 2023. During this time, LHC will be out of operation for furnishing with new inner triplets and crab cavities. As a result, an instantaneous luminosity of $5 \times 10^{34} \mathrm{~cm}^{-2} \mathrm{~s}^{-1}$ should be achieved. The goal is to accumulate $3000 \mathrm{fb}^{-1}$ of data by $\sim 2030$.

ATLAS Phase-II preparations include a new Inner Detector and further trigger and calorimeter upgrades.

\subsection{New Inner Detector}

Running at nominal $\mathcal{L}^{\text {peak }}$ for the LHC, will bring, on average, $\sim 28$ primary interactions (pile-up events) per bunch crossing, every $25 \mathrm{~ns}$. The number of pile-up events at $5 \times 10^{34} \mathrm{~cm}^{-2} \mathrm{~s}^{-1}$ is therefore expected to be $\sim 140$. (Should luminosity levelling not be fully effective or some other scheme adopted, $7 \times 10^{34} \mathrm{~cm}^{-2} \mathrm{~s}^{-1}$ should at least be accommodated.) This will result in 5 to 10 times higher detector occupancies, which is beyond the TRT design parameters. Furthermore, by 2022, the Pixel and the SCT subsystems, would seriously degrade their performance due to the radiation damage of their sensors and FE electronics. Because of all these factors, ATLAS has decided to replace the entire Inner Detector with a new, all-silicon Inner Tracker (ITk). The ITk must satisfy the following criteria (w.r.t. ID): higher granularity, improved material budget, increased radiation resistivity of the readout components. At the moment, the ITk project is in an R\&D phase. Different geometrical layouts are simulated and their performance is studied in search for the optimal tracker architecture. A major constraint on the design is the available space, defined by the volume taken by the ID in ATLAS. This implies a maximum radius of $\sim 1 \mathrm{~m}$ and the limiting existing gaps for services.

The current baseline design of the ITk, depicted in Fig. 3 , consists of 4 Pixel and $5 \mathrm{Si}$-strip layers in the barrel part. The two endcap regions are each composed of 6 Pixel and 5 Si-strip double-sided disks, built of rings of modules. The pixel modules are with identical pixels of size $50 \times 250 \mu \mathrm{m}$, whereas the Si-strip modules come in two types, with short (24 $\mathrm{mm}$ ) and long $(96 \mathrm{~mm})$ strips. As in the current SCT, the Si-strip modules are designed to be of 2 pairs of silicon microstrip sensors, glued back-to-back at an angle of 40 mrad to provide $2 \mathrm{D}$ space-points.

Intensive R\&D studies are also in process to select the most suitable pixel sensor technology out of Si-planar, 3D and diamond, and to find the optimal layout of the Si-strip modules [8].

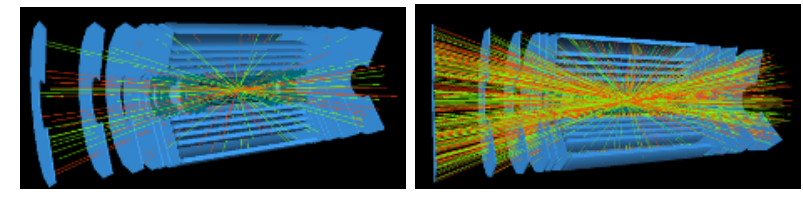

Fig. 3. The baseline layout of the new Inner Detector, traversed by simulated 23 pile-up events (left) and 230 pile-up events (right).

\subsection{Calorimeter and trigger upgrades}

The HL-LHC conditions will have a major impact on the Calorimetry system. To ensure an adequate performance, a replacement of the cold electronics inside the LAr Hadronic endcap, as well as, a replacement of all on-detector readout electronics for all calorimeters may need to be anticipated. Also, the operation of the Forward Calorimeter (FCal) could be compromised. To maintain the FCal functioning at the HL-LHC, two possible solutions are considered [7]: first, complete replacement of the FCal, and second, installation of a small warm calorimeter, Mini-FCal, in front of the FCal. The Mini-Fcal would reduce the ionization and heat loads of the FCal to acceptable levels.

The planned trigger upgrades for Phase-II, are connected with implementing a Track Trigger at Level-1/Level2 , applying full granularity of calorimeter at Level-1 and improving the muon trigger coverage.

\section{Conclusions}

ATLAS collaboration has devised a detailed program to reflect the changes in the LHC conditions towards the HighLuminosity LHC, characterized by high track multiplicity and extreme fluences. At each of the 3 phases of the upgrade program, actions will be undertaken to reassure the stable and efficient performance of the ATLAS detector.

\section{References}

1. G. Aad, et al., The ATLAS Experiment at the CERN Large Hadron Collider, J. Instr. 3 (2008) S08003.

2. L. Evans and P. Bryant, LHC Machine, J. Instr. 3 (2008) S08001.

3. K. Jakobs, Physics at the LHC and $S L H C$, Nucl. Instr. and Meth. A (2010), doi:10.1016/j.nima.2010.04.077

4. ATLAS collaboration, Insertable B-Layer, Technical Design Report, CERN/LHCC-2010-013

5. M. Barbero et al., A new ATLAS pixel front-end IC for upgraded LHC luminosity, Nucl. Instrum. Meth. A 604 (2009) 397.

6. M.S. Neubauer, A Fast Hardware Tracker for the ATLAS Trigger System, ATL-DAQ-PROC-2011-023, arXiv:1110.1910v1 [hep-ex]

7. J. Turner, Upgrade Plans for ATLAS Forward Calorimetry for the HL-LHC, ATL-LARG-PROC-2011002

8. A. Affolder, Silicon Strip Detectors for the ATLAS HLLHC Upgrade, ATL-UPGRADE-PROC-2011-005

9. B. Bittner, et al., Tracking and Level-1 triggering in the forward region of the ATLAS Muon Spectrometer at $s L H C$, ATL-UPGRADE-PROC-2011-008 